\section{In Vivo Calcium Imaging to Illuminate Neurocircuit Activity Dynamics Underlying Naturalistic Behavior}

The brain perceives the outside world, coordinates behavior, regulates emotional states, and forms memories about these experiences. To orchestrate such a diverse array of functions, billions of neurons interact to form circuits with the capacity to compute sensory infor- mation and rapidly generate appropriate behavior. Understanding these processes and how the dysregulation of circuits contributes to psychiatric illness is a major goal of neuroscience research and has led to the development of novel tools for circuit-level analysis of the a

In vivo Microendoscopy

$D A Q$ box

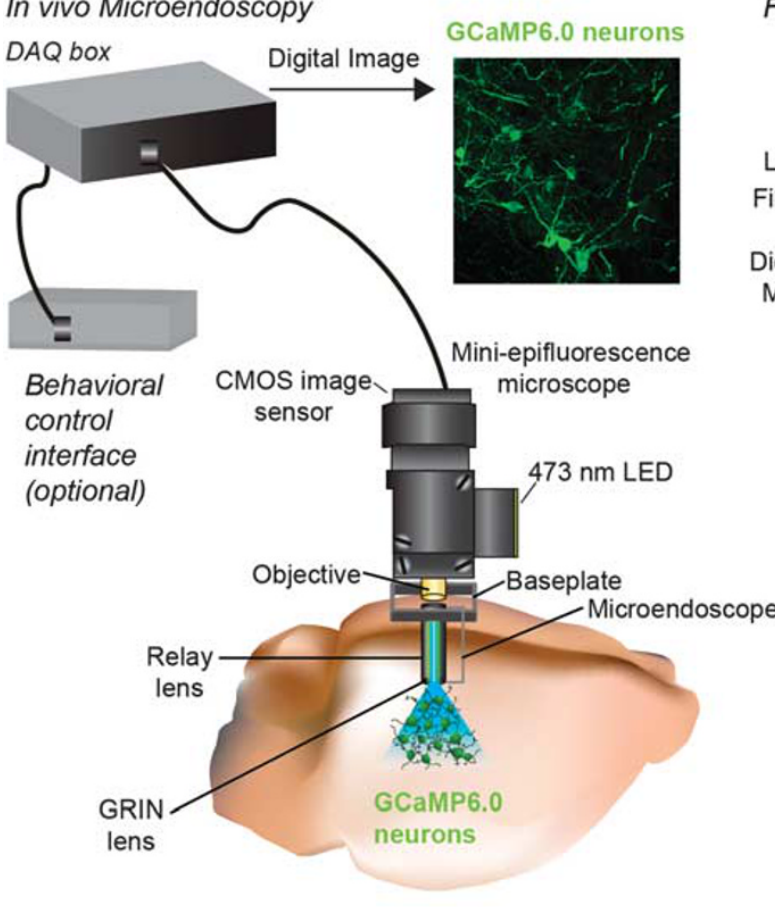

b

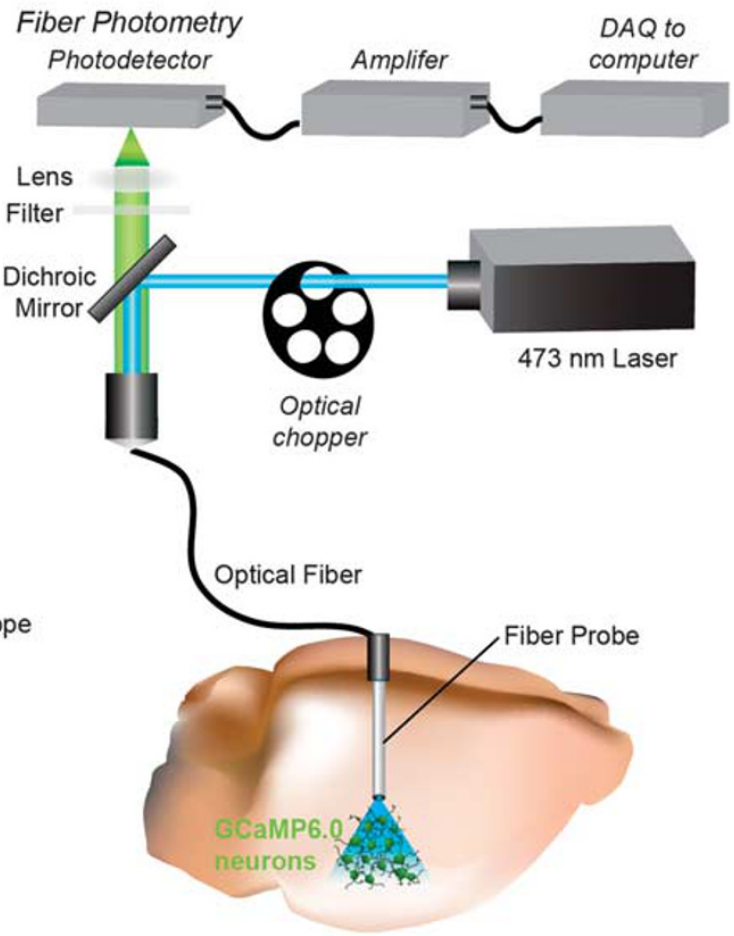

C

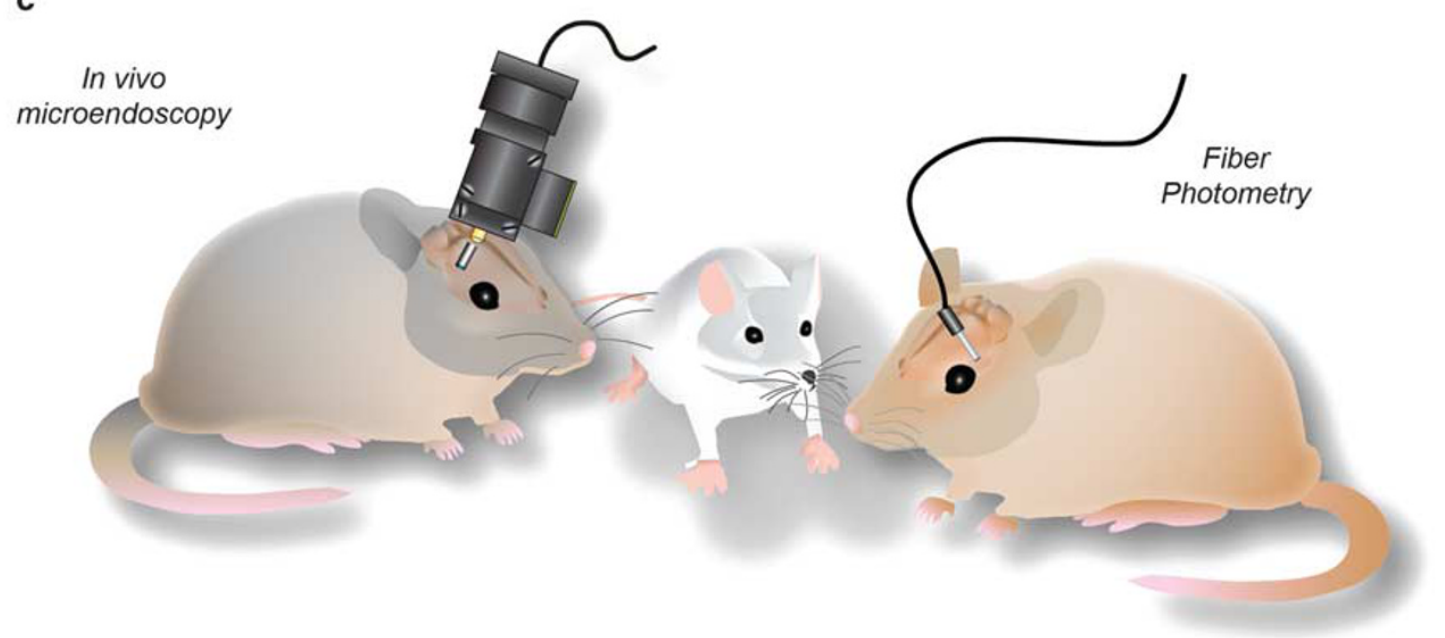

Figure 1. Optical technologies for in vivo calcium imaging in freely behaving mice. (a) Mini-epifluorescence microscope attached to a head-mounted base plate that is centered around a microendoscope implanted above a brain region of interest. (b) A fiber probe is implanted above the target brain region of interest to collect bulk changes in calcium-mediated fluorescence using fiber photometry. (c) Animals engaging in naturalistic behavior such as social interaction while fluorescent transients are measured as a read-out of neural activity with the use of microendoscopic imaging (left) or fiber photometry (right). 
brain in behaving animals (Jennings and Stuber, 2014). One approach for monitoring circuit-specific activity dynamics is in vivo calcium imaging. During periods of increased neural activity, dynamic fluctuations in calcium levels correlate with events such as action potential generation, exocytosis of neurotransmitters, changes in synaptic plasticity, and gene transcription. Thus, monitoring calcium dynamics with genetically encoded calcium indicators, such as GCaMP6, can serve as a proxy for neural activity within defined neurocircuits (Chen et al, 2013). Imaging of neural activity using calcium indicators has largely been accomplished with in vivo two-photon microscopy, which provides unprecedented cellular and subcellular spatial resolution; however, two-photon imaging must be performed in head-fixed animals, greatly limiting the assessment of naturalistic behavior (Svoboda and Yasuda, 2006). To circumvent these limitations, new methods for visualizing and quantifying calciummediated fluorescent signals have been developed for use in freely moving animals.

Visualizing calcium transients in freely moving mice requires imaging devices that are small enough to fit on an animal's head and light enough to be carried by the animal. In the last few years, two such approaches have been developed: mini-epifluorescence microscopes used in conjunction with gradient index (GRIN) lens microendoscopes (Ziv et al, 2013) and fiber photometry (Cui et al, 2013; Gunaydin et al, 2014) (Figure 1). Mini-epifluorescence microscopes are ideal for measuring somatic calcium activity dynamics. Importantly, with this novel technology, the activity of hundreds of genetically and spatially defined neurons within a single animal can be repeatedly imaged, permitting the analysis of cellular network dynamics that orchestrate behavior as well as the transition to pathological disease states (Ziv et al, 2013). In addition, both microendoscopic imaging and fiber photometry are capable of recording neural activity many millimeters deep within the brain. Fiber photometry utilizes optical fibers to detect bulk changes in calcium-mediated fluorescence in the soma (Cui et al, 2013) or terminal fields (Gunaydin et al, 2014) of genetically defined neurons. While fiber photometry lacks cellular resolution, it can provide important insight into the synchronous activity dynamics within a defined neurocircuit during both adaptive and aberrant behavioral states. Importantly, the light weight and small size of both these devices allows them to be coupled with a wide array of behavioral paradigms, such as sensory tasks, elevated plus maze for measures of anxiety, tail suspension for research related to depression, as well as complex learning paradigms utilizing operant chambers. Although advances are continuously being made in all aspects of these devices, current models of the mini-epifluorescence microscopes are not yet compatible with behavioral paradigms where mice are submerged in water, such as the forced swim test. Nonetheless, these innovative approaches can elucidate the temporal and spatial activity patterns of molecularly defined neurocircuits during a multitude of adaptive and maladaptive behaviors.

\section{ACKNOWLEDGEMENTS}

We thank Joshua Jennings for assistance in preparing the figure. We thank the Brain and Behavior Research Foundation, The Foundation of Hope, The Klarman Family Foundation, the National Institute on Drug Abuse (DA032750, DA038168) (GDS), and the Carolina Institute for Developmental Disabilities (T32HD040127) (SLR) for support.

\section{Shanna L Resendez ${ }^{1,2}$ and \\ Garret D Stuber ${ }^{1,2}$ \\ ${ }^{1}$ Departments of Psychiatry \& Cell and Molecular Physiology, UNC Neuroscience Center, University of North Carolina at Chapel Hill, Chapel Hill, NC, USA; ${ }^{2}$ Neuroscience Center, University of North Carolina at Chapel Hill, Chapel Hill, NC, USA \\ E-mail: gstuber@med.unc.edu}

\section{FUNDING AND DISCLOSURE}

G.D. Stuber has received prototype versions of a mini-epifluorescence microscope system from Inscopix Inc. for testing purposes. The authors declare no conflict of interest.

Chen T-W, Wardill TJ, Sun Y, Pulver SR, Renninger SL, Baohan A et al. (2013). Ultrasensitive fluorescent proteins for imaging neuronal activity. Nature 499 : 295-300.

Cui G, Jun SB, Jin X, Pham MD, Vogel SS, Lovinger $\mathrm{DM}$ et al. (2013). Concurrent activation of striatal direct and indirect pathways during action initiation. Nature 494: 238-242.

Gunaydin LA, Grosenick L, Finkelstein JC, Kauvar IV, Fenno LE, Adhikari A et al. (2014). Natural neural projection dynamics underlying social behavior. Cell 157: 1535-1551.

Jennings JH, Stuber GD (2014). Tools for resolving functional activity and connectivity within intact neural circuits. Curr Biol 24: R41-R50.

Svoboda K, Yasuda R (2006). Principles of twophoton excitation microscopy and its applications to neuroscience. Neuron 50: 823-839.

Ziv Y, Burns LD, Cocker ED, Hamel EO, Ghosh KK, Kitch LJ et al. (2013). Long-term dynamics of CA1 hippocampal place codes. Nat Neurosci 16: 264-266.

Neuropsychopharmacology Reviews (2015) 40, 238-239; doi: I0.1038/npp.20|4.206

\section{DREAMM: A Biobehavioral Imaging Methodology for Dynamic In Vivo Whole-Brain Mapping of Cell Type-Specific Functional Networks}

The human brain contains $\sim 100$ billion anatomically and neurochemically heterogeneous neurons that form 100 trillion brain-wide connections giving rise to a complex network whose interconnected activity regulates behavior. A major challenge in neuroscience has been to decipher the discrete functional connectivity underlying normal or pathological behavior.

Until recently, the primary methods for determining whole-brain functional activity as a consequence of discrete manipulation of specific cell populations was to study indirect markers such as c-Fos immunoreactivity in postmortem sections cut throughout the brain, or via in vivo blood oxygenation level-dependent measures using functional magnetic resonance imaging (fMRI), which requires experimental subjects to be fully immobilized. In an effort to overcome such limitations, we recently described the development and application of a new biobehavioral imaging methodology termed DREADD-assisted metabolic 